\title{
Limited Feedback for Interference Alignment in the K-user MIMO Interference Channel
}

\author{
Mohsen Rezaee, Maxime Guillaud \\ Institute of Telecommunications, Vienna University of Technology, \\ Vienna, Austria - e-mail: \{guillaud, mohsen.rezaee\}@tuwien.ac.at
}

\begin{abstract}
A simple limited feedback scheme is proposed for interference alignment on the K-user Multiple-Input-MultipleOutput Interference Channel (MIMO-IC). The scaling of the number of feedback bits with the transmit power required to preserve the multiplexing gain that can be achieved using perfect channel state information (CSI) is derived. This result is obtained through a reformulation of the interference alignment problem in order to exploit the benefits of quantization on the Grassmann manifold, which is well investigated in the single-user MIMO channel. Furthermore, through simulations we show that the proposed scheme outperforms the naive feedback scheme consisting in independently quantizing the channel matrices, in the sense that it yields a better sum rate performance for the same number of feedback bits.
\end{abstract}

\section{INTRODUCTION}

Multiple-antenna transceivers improve the performance of a wireless communication link compared to single-antenna systems. The increasing demand for high throughput and reliable transmission necessitates efficient use of MultipleInput-Multiple-Output (MIMO) systems. The availability of channel state information (CSI) at the transmitter is crucial in order to fully exploit the performance improvement of MIMO systems.

In scenarios where the channel is not reciprocal (such as frequency-division duplex systems) the CSI has to be quantized and fed back to the transmitter. The mismatch between the true channel and the quantized channel results in a degradation in performance. This degradation is more severe when it comes to multi-user systems because the channel mismatch not only reduces the effective channel gain but also causes interference for the other users.

Interference alignment (IA) is a precoding method that achieves the optimal multiplexing gain (also called the degrees of freedom, DoF) over the $K$-user interference channel when perfect CSI is available at the transmitters [1]. This method designs the precoders such that the total interference at each receiver lies in a space with minimum dimensions so that the rest of the dimensions can be used for interference-free decoding. IA has been introduced for the $K$-user MIMO IC in [2].

Extensive research has been made on limited feedback schemes for single-user MIMO systems [3, and references therein]. In [4], codebook design is investigated when the receiver selects the best unitary precoder from a finite codebook and feeds back the index of the selected precoder to the transmitter. [4] shows that the optimal design for such a codebook is equivalent to the Grassmannian subspace packing problem. Some useful quantization bounds on the Grassmann manifold are derived in [5], [6]. In [7], quantization of the precoding matrix using a random vector quantization (RVQ) codebooks is investigated which provides insights on the asymptotic optimality of RVQ codebooks.

In the interference channel the problem is explored in the context of interference alignment over frequency selective channels for single-antenna users in [8] and for multipleantenna users in [9]. Both references provide DoF-achieving quantization schemes and study the required scaling of the number of feedback bits. From another point of view, [10] provides an analysis of the effect of imperfect CSI on the mutual information of the interference alignment scheme. The authors in [11] proposed a method to reduce the quantization error w.r.t. the classical scheme; the method involves a computationally heavy iterative algorithm which must be ran for each codeword and for each channel realization.

In this paper we present a simple feedback scheme based on a Grassmannian representation of CSI and we characterize the proper scaling of the number of feedback bits with the transmit power in order to preserve the multiplexing gain achievable under perfect CSI. Using the structure of the interference alignment equations, we remove the redundant information in the channel quantization procedure. Simulations are also provided to compare our proposed method to existing feedback methods.

The remainder of the paper is organized as follows. In Section II, the system model is described. The reformulation of the interference alignment problem is provided in Section III. The limited feedback method is presented in Section IV. Simulation results are presented in Section $\mathrm{V}$ and conclusions are drawn in Section VI.

Notation: Non-bold letters represent scalar quantities, boldface lowercase and uppercase letters indicate vectors and matrices, respectively. $\mathbf{I}_{N}$ is the $N \times N$ identity matrix. The trace, conjugate, transpose, Hermitian transpose of a matrix or vector are denoted by $\operatorname{tr}(\cdot),(\cdot)^{*},(\cdot)^{\mathrm{T}},(\cdot)^{\mathrm{H}}$ respectively. The expectation operator is represented by $\mathrm{E}(\cdot)$. The determinant of a matrix (or absolute value of a scalar) is represented by $|\cdot|$. The Frobenius norm of a matrix is denoted by $\|\cdot\|_{F}$ while the two-norm (spectral norm) of a matrix is represented by 
$\|\cdot\|_{2}$. A block diagonal matrix is denoted by $\operatorname{Bdiag}(\cdot)$ with the argument blocks on its diagonal. $\mathcal{C N}(0,1)$ denotes the complex Gaussian circularly symmetric distribution with zero mean and unit variance. Finally, log represents the logarithm in base 2 .

\section{SySTEM MOdEL}

An interference channel is considered in which $K$ transmitters communicate with their respective receivers over a shared medium. For the sake of notational simplicity, we consider the symmetric case where each transmitter has $M$ antennas while each receiver is equipped with $N$ antennas, although the method applies to more general non-symmetric settings as well.

Each transmitter employs a precoding matrix to transmit $d$ data streams to its respective receiver. The vector at receiver $i$ reads

$$
\mathbf{y}_{i}=\mathbf{H}_{i i} \mathbf{V}_{i} \mathbf{x}_{i}+\sum_{\substack{1 \leq j \leq K \\ j \neq i}} \mathbf{H}_{i j} \mathbf{V}_{j} \mathbf{x}_{j}+\mathbf{n}_{i}
$$

in which $\mathbf{H}_{i j} \in \mathbb{C}^{N \times M}$ is the channel matrix between transmitter $j$ and receiver $i, \mathbf{V}_{j} \in \mathbb{C}^{M \times d}$ and $\mathbf{x}_{j} \in \mathbb{C}^{d}$ are the precoding matrix and the data vector of transmitter $j$, respectively. Furthermore, $\mathbf{n}_{i} \sim \mathcal{C N}\left(0, \sigma^{2} \mathbf{I}_{N}\right)$ is the additive noise at receiver $i$. Assuming $\mathrm{E}\left[\mathbf{x}_{j} \mathbf{x}_{j}^{\mathrm{H}}\right]=\frac{P}{d} \mathbf{I}_{d}, j=1, \ldots, K$ and using truncated unitary precoders, the transmit power for each user is equal to $P$. The transmit signal-to-noise ratio (SNR) is defined as $\frac{P}{\sigma^{2}}$.

\section{GRASSmannian FeEdback FOR IA}

Let us consider the interference alignment problem of [2], in which the channel state information is fed back from the receivers to the transmitters and the transmitters find the precoding and projecting matrices that satisfy the alignment conditions based on the received feedback. This scenario arises when the receivers cannot share their channel estimates. The $i$ th receiver estimates the channel matrices $\mathbf{H}_{i j}, j \neq i$ and feeds back the necessary information to all the transmitters so that every transmitter is capable of solving the alignment problem. In this section we consider perfect CSI feedback in order to to highlight the intuition behind our limited feedback scheme.

Assuming that global CSI is available at a given location, the precoders $\mathbf{V}_{i}, i=1 \ldots K$ can be designed to align the interference at each receiver into a $N-d$ dimensional subspace. Therefore, interference-free transmission of $d$ streams per user becomes possible for a feasible setting. We will further assume that $(K-1) M \geq N$, which represents the cases of interest where interference would occupy all dimensions of the receive subspace in the absence of alignment. A solution to the IA problem exists (see [12] and more recently [13], [14] for feasibility criteria - here we will assume that the dimensions and the considered channel realizations are such that the problem is feasible) iff there exist full rank precoding matrices $\mathbf{V}_{j}, j=1, \ldots, K$ and projection matrices $\mathbf{U}_{i} \in \mathbb{C}^{N \times d}, i=1, \ldots, K$ such that

$$
\begin{aligned}
& \mathbf{U}_{i}^{\mathrm{H}} \mathbf{H}_{i j} \mathbf{V}_{j}=\mathbf{0} \quad \forall i, j \in\{1, \ldots, K\}, j \neq i, \\
& \operatorname{rank}\left(\mathbf{U}_{i}^{\mathrm{H}} \mathbf{H}_{i i} \mathbf{V}_{i}\right)=d .
\end{aligned}
$$

The first condition can be written from the point of view of receiver $i$ in the form

$$
\mathbf{U}_{i}^{\mathrm{H}} \mathbf{H}_{i} \mathbf{V}_{-i}=\mathbf{0} \quad \forall i \in\{1, \ldots, K\}
$$

in which $\mathbf{V}_{-i}=\operatorname{Bdiag}\left(\mathbf{V}_{1}, \ldots, \mathbf{V}_{i-1}, \mathbf{V}_{i+1}, \ldots, \mathbf{V}_{K}\right)$ and $\mathbf{H}_{i}=\left[\mathbf{H}_{i 1}, \ldots, \mathbf{H}_{i i-1}, \mathbf{H}_{i i+1}, \ldots, \mathbf{H}_{i K}\right]$ which is a $N \times(K-$ 1) $M$ matrix. We consider the thin QR decomposition of $\mathbf{H}_{i}^{\mathrm{H}}$, defined as $\mathbf{H}_{i}^{\mathrm{H}}=\mathbf{F}_{i} \mathbf{C}_{i}$ in which $\mathbf{F}_{i}$ is a $(K-1) M \times N$ truncated unitary matrix and $\mathbf{C}_{i}$ is a $N \times N$ matrix. Note that in many cases of interest, such as when the channel matrices have coefficients independently drawn from a continuous distribution, $\mathbf{H}_{i}$ is full row rank with probability 1 , and therefore $\mathbf{C}_{i}$ is invertible almost surely (a.s.). If we employ the filter $\left(\mathbf{C}_{i}^{-1}\right)^{\mathrm{H}}$ at the $i$ th receiver before projecting the signal into the interference-free subspace then we can solve the alignment problem at the transmitters based on $\mathbf{F}_{i}^{\mathrm{H}}$ instead of $\mathbf{H}_{i}$, since

$$
\begin{aligned}
\mathbf{U}_{i}^{\mathrm{H}} \mathbf{F}_{i}^{\mathrm{H}} \mathbf{V}_{-i}=\mathbf{0} & \Rightarrow \mathbf{U}_{i}^{\mathrm{H}} \mathbf{C}_{i}^{-\mathrm{H}} \mathbf{C}_{i}^{\mathrm{H}} \mathbf{F}_{i}^{\mathrm{H}} \mathbf{V}_{-i}=\mathbf{0} \\
& \Rightarrow\left(\mathbf{C}_{i}^{-1} \mathbf{U}_{i}\right)^{\mathrm{H}} \mathbf{H}_{i} \mathbf{V}_{-i}=\mathbf{0} .
\end{aligned}
$$

This indicates that if the transmitters design $\left(\left\{\mathbf{V}_{i}\right\}_{i=1}^{K},\left\{\mathbf{U}_{i}\right\}_{i=1}^{K}\right)$ for $\left\{\mathbf{F}_{i}^{\mathrm{H}}\right\}_{i=1}^{K}$, then we can suppress the interference at receiver $i$ using the $\mathbf{V}_{i}$ 's as precoders and $\left(\mathbf{C}_{i}^{-1} \mathbf{U}_{i}\right)^{\mathrm{H}}$ as the projection filter at receiver $i$.

The importance of this observation is that $\mathbf{F}_{i}$ is a truncated unitary matrix and its structure offers a reduction in the amount of required feedback. We now show that the knowledge of the column space of $\mathbf{F}_{i}$ is sufficient to compute precoding matrices that fulfill the alignment conditions.

Considering perfect CSI feedback on the Grassmann manifold, the feedback can be considered to take the form of a matrix whose columns span the same subspace as those of $\mathbf{F}_{i}$, and which can be written as $\mathbf{F}_{i} \overline{\mathbf{U}}_{i}$ with $\overline{\mathbf{U}}_{i}$ being a unitary matrix. The transmitter finds the alignment solution based on $\mathbf{F}_{i} \overline{\mathbf{U}}_{i}$. Therefore we have

$$
\tilde{\mathbf{U}}_{i}^{\mathrm{H}}\left(\mathbf{F}_{i} \overline{\mathbf{U}}_{i}\right)^{\mathrm{H}} \mathbf{V}_{-i}=\mathbf{0} \Rightarrow\left(\overline{\mathbf{U}}_{i} \tilde{\mathbf{U}}_{i}\right)^{\mathrm{H}} \mathbf{F}_{i}^{\mathrm{H}} \mathbf{V}_{-i}=\mathbf{0} .
$$

Letting $\mathbf{U}_{i}=\overline{\mathbf{U}}_{i} \tilde{\mathbf{U}}_{i}$ and using the same argument as in (4), the interference can be completely removed by using the filter $\left(\mathbf{C}_{i}^{-1} \mathbf{U}_{i}\right)^{\mathrm{H}}=\left(\mathbf{C}_{i}^{-1} \overline{\mathbf{U}}_{i} \tilde{\mathbf{U}}_{i}\right)^{\mathrm{H}}$ at receiver $i$. This means that the receiver should rotate the alignment projection matrix $\left(\tilde{\mathbf{U}}_{i}\right)$ using the same rotation employed for feedback and then transform it by $\mathbf{C}_{i}^{-1}$. Assuming that the process of reconstructing $\mathbf{F}_{i} \overline{\mathbf{U}}_{i}$ from the fed back message is deterministic and known to the receiver, $\mathbf{F}_{i} \overline{\mathbf{U}}_{i}$ and therefore $\overline{\mathbf{U}}_{i}$ can be assumed to be known at the receiver. $\tilde{\mathbf{U}}_{i}$, however, is not known at the receiver and must be communicated or evaluated through other means.

It is obvious from (4) that the CSI feedback problem in this context is equivalent to feeding back a point (represented by the truncated unitary matrix $\mathbf{F}_{i}$ ) on the Grassmann manifold $\mathcal{G}_{(K-1) M, N}$ for each of the $\mathrm{K}$ users. 


\section{QuAntized CSI FEEDbACK Scheme}

In this section we consider the case where the alignment equations are solved based on the (error-free) feedback of a quantized version of the CSI, using the Grassmannian representation outlined in the previous section. Let us assume that receiver $i$ perfectly knows its channels from all interfering transmitters and therefore can perform the QR decomposition $\mathbf{H}_{i}^{\mathrm{H}}=\mathbf{F}_{i} \mathbf{C}_{i}$. Receiver $i$ quantizes the subspace spanned by the columns of $\mathbf{F}_{i}$ using $N_{f}$ bits and broadcasts the index of the quantized codeword to the transmitters or any other processing unit in charge of computing the $\mathbf{V}_{i}$ 's. We further assume that the transmitters and receivers share a predefined codebook $\mathcal{S}=\left\{\mathbf{S}_{1}, \ldots, \mathbf{S}_{2^{N_{f}}}\right\}$ which is composed of $2^{N_{f}}$ truncated unitary matrices of size $(K-1) M \times N$ and ideally is designed using Grassmannian subspace packing. The quantized codeword is the closest point on the Grassmann manifold, i.e.,

$$
\hat{\mathbf{F}}_{i}=\arg \min _{\mathbf{S}_{x} \in \mathcal{S}} d_{c}\left(\mathbf{S}_{x}, \mathbf{F}_{i}\right)
$$

in which $d_{c}(\mathbf{X}, \mathbf{Y})=\frac{1}{\sqrt{2}}\left\|\mathbf{X} \mathbf{X}^{H}-\mathbf{Y} \mathbf{Y}^{H}\right\|_{\mathbf{F}}$ is the chordal distance between $\mathbf{X}$ and $\mathbf{Y} \in \mathcal{G}_{(K-1) M, N}$ [15].

The interference alignment problem is then solved based on $\left\{\hat{\mathbf{F}}_{i}^{\mathrm{H}}\right\}_{i=1}^{K}$ to find $\left(\left\{\mathbf{V}_{i}\right\}_{i=1}^{K},\left\{\tilde{\mathbf{U}}_{i}\right\}_{i=1}^{K}\right)$ fulfilling

$$
\tilde{\mathbf{U}}_{i}^{\mathrm{H}} \hat{\mathbf{F}}_{i}^{\mathrm{H}} \mathbf{V}_{-i}=\mathbf{0}, \quad \forall i \in\{1, \ldots, K\} .
$$

At receiver $i$, let us define the (not necessarily unitary) matrix $\breve{\mathbf{U}}_{i}=\mathbf{F}_{i}^{\mathrm{H}} \hat{\mathbf{F}}_{i}$. Furthermore, inspired by the perfect feedback situation, we define the total receive filter as $\mathbf{G}_{i}^{\mathrm{H}}=\left(\mathbf{C}_{i}^{-1} \breve{\mathbf{U}}_{i} \tilde{\mathbf{U}}_{i}\right)^{\mathrm{H}}=\left(\mathbf{C}_{i}^{-1} \mathbf{F}_{i}^{\mathrm{H}} \hat{\mathbf{F}}_{i} \tilde{\mathbf{U}}_{i}\right)^{\mathrm{H}}$. It should be noted that when $\mathbf{F}_{i}$ lies in the subspace of one of the codebook elements (i.e., $d_{c}\left(\hat{\mathbf{F}}_{i}, \mathbf{F}_{i}\right)=0$ ) then $\breve{\mathbf{U}}_{i}$ becomes a unitary matrix and will be equal to $\overline{\mathbf{U}}_{i}$ from Section III.

We now show that our limited feedback scheme preserves the total multiplexing gain of the channel provided that the number of feedback bits grows with the transmit power, and we characterize the required growth rate.

After applying the receive filter $\mathbf{G}_{i}^{\mathrm{H}}$ to (1), the interference leakage (due to imperfect CSI) at receiver $i$ can be written as

$$
\mathbf{e}_{i}=\sum_{\substack{1 \leq j \leq K \\ j \neq i}} \mathbf{G}_{i}^{\mathrm{H}} \mathbf{H}_{i j} \mathbf{V}_{j} \mathbf{x}_{j} .
$$

Therefore the power of the interference leakage at receiver $i$ reads

$$
\begin{aligned}
L_{i} & =\operatorname{tr}\left(\mathrm{E}_{\mathbf{x}}\left(\mathbf{e}_{i} \mathbf{e}_{i}^{\mathrm{H}}\right)\right) \\
& =\operatorname{tr}\left(\frac{P}{d} \sum_{j=1, j \neq i}^{K} \mathbf{G}_{i}^{\mathrm{H}} \mathbf{H}_{i j} \mathbf{V}_{j} \mathbf{V}_{j}^{\mathrm{H}} \mathbf{H}_{i j}^{\mathrm{H}} \mathbf{G}_{i}\right) \\
& =\frac{P}{d} \operatorname{tr}\left(\mathbf{G}_{i}^{\mathrm{H}} \mathbf{H}_{i} \mathbf{V}_{-i} \mathbf{V}_{-i}^{\mathrm{H}} \mathbf{H}_{i}^{\mathrm{H}} \mathbf{G}_{i}\right) \\
& =\frac{P}{d}\left\|\mathbf{G}_{i}^{\mathrm{H}} \mathbf{H}_{i} \mathbf{V}_{-i}\right\|_{\mathrm{F}}^{2} .
\end{aligned}
$$

Substituting $\mathbf{G}_{i}^{\mathrm{H}}=\left(\mathbf{C}_{i}^{-1} \mathbf{F}_{i}^{\mathrm{H}} \hat{\mathbf{F}}_{i} \tilde{\mathbf{U}}_{i}\right)^{\mathrm{H}}$ and $\mathbf{H}_{i}=\mathbf{C}_{i}^{\mathrm{H}} \mathbf{F}_{i}^{\mathrm{H}}$ gives

$$
\begin{aligned}
L_{i} & =\frac{P}{d}\left\|\tilde{\mathbf{U}}_{i}^{\mathrm{H}} \hat{\mathbf{F}}_{i}^{\mathrm{H}} \mathbf{F}_{i} \mathbf{C}_{i}^{-\mathrm{H}} \mathbf{C}_{i}^{\mathrm{H}} \mathbf{F}_{i}^{\mathrm{H}} \mathbf{V}_{-i}\right\|_{\mathrm{F}}^{2} \\
& =\frac{P}{d}\left\|\tilde{\mathbf{U}}_{i}^{\mathrm{H}} \hat{\mathbf{F}}_{i}^{\mathrm{H}} \mathbf{F}_{i} \mathbf{F}_{i}^{\mathrm{H}} \mathbf{V}_{-i}\right\|_{\mathrm{F}}^{2} .
\end{aligned}
$$

Using the alignment equation in (7) and the fact that $\hat{\mathbf{F}}_{i}^{\mathrm{H}} \hat{\mathbf{F}}_{i}=$ $\mathbf{I}_{N}$, yields $\tilde{\mathbf{U}}_{i}^{\mathrm{H}} \hat{\mathbf{F}}_{i}^{\mathrm{H}} \hat{\mathbf{F}}_{i} \hat{\mathbf{F}}_{i}^{\mathrm{H}} \mathbf{V}_{-i}=0$, therefore (10) can be rewritten as

$$
L_{i}=\frac{P}{d}\left\|\tilde{\mathbf{U}}_{i}^{\mathrm{H}} \hat{\mathbf{F}}_{i}^{\mathrm{H}}\left(\mathbf{F}_{i} \mathbf{F}_{i}^{\mathrm{H}}-\hat{\mathbf{F}}_{i} \hat{\mathbf{F}}_{i}^{\mathrm{H}}\right) \mathbf{V}_{-i}\right\|_{\mathrm{F}}^{2} .
$$

Using the facts that $\|\mathbf{X}\|_{\mathrm{F}} \leq \sqrt{\operatorname{rank}(\mathbf{X})} \cdot\|\mathbf{X}\|_{2},\|\mathbf{X}\|_{2} \leq$ $\|\mathbf{X}\|_{\mathrm{F}}$ and $\|\mathbf{X Y}\|_{2} \leq\|\mathbf{X}\|_{2}\|\mathbf{Y}\|_{2}$, we have

$$
\begin{aligned}
L_{i} & =\frac{P}{d}\left\|\tilde{\mathbf{U}}_{i}^{\mathrm{H}} \hat{\mathbf{F}}_{i}^{\mathrm{H}}\left(\mathbf{F}_{i} \mathbf{F}_{i}^{\mathrm{H}}-\hat{\mathbf{F}}_{i} \hat{\mathbf{F}}_{i}^{\mathrm{H}}\right) \mathbf{V}_{-i}\right\|_{\mathrm{F}}^{2} \\
& \leq P\left\|\tilde{\mathbf{U}}_{i}^{\mathrm{H}} \hat{\mathbf{F}}_{i}^{\mathrm{H}}\left(\mathbf{F}_{i} \mathbf{F}_{i}^{\mathrm{H}}-\hat{\mathbf{F}}_{i} \hat{\mathbf{F}}_{i}^{\mathrm{H}}\right) \mathbf{V}_{-i}\right\|_{2}^{2} \\
& \leq P\left\|\tilde{\mathbf{U}}_{i}^{\mathrm{H}}\right\|_{2}^{2}\left\|\hat{\mathbf{F}}_{i}^{\mathrm{H}}\right\|_{2}^{2}\left\|\left(\mathbf{F}_{i} \mathbf{F}_{i}^{\mathrm{H}}-\hat{\mathbf{F}}_{i} \hat{\mathbf{F}}_{i}^{\mathrm{H}}\right)\right\|_{2}^{2}\left\|\mathbf{V}_{-i}\right\|_{2}^{2} \\
& =P\left\|\left(\mathbf{F}_{i} \mathbf{F}_{i}^{\mathrm{H}}-\hat{\mathbf{F}}_{i} \hat{\mathbf{F}}_{i}^{\mathrm{H}}\right)\right\|_{2}^{2} \\
& \leq P\left\|\left(\mathbf{F}_{i} \mathbf{F}_{i}^{\mathrm{H}}-\hat{\mathbf{F}}_{i} \hat{\mathbf{F}}_{i}^{\mathrm{H}}\right)\right\|_{\mathrm{F}}^{2} \\
& =2 P d_{c}^{2}\left(\hat{\mathbf{F}}_{i}, \mathbf{F}_{i}\right) .
\end{aligned}
$$

The second equality holds because $\tilde{\mathbf{U}}_{i}^{\mathrm{H}}, \hat{\mathbf{F}}_{i}^{\mathrm{H}}$ and each block of $\mathbf{V}_{-i}$ are truncated unitary matrices which implies that the two-norm of these matrices is 1 .

Using [5, Corollary 1] and [15, Theorem 5], if the codebook is generated using sphere-packing procedure, the maximum value of quantization error in terms of the chordal distance can be upper bounded as

$$
\Delta_{i \max } \leq \frac{2}{\left(c 2^{N_{f}}\right)^{\frac{1}{N_{G}}}}
$$

in which $\Delta_{i \max }=\max _{\mathbf{F}_{i} \in \mathcal{G}_{(K-1) M, N}} d_{c}\left(\hat{\mathbf{F}}_{i}, \mathbf{F}_{i}\right)$, the constant $c$ is the coefficient of the ball volume in the Grassmann manifold which is given in [5, Theorem 1] and $N_{G}=$ $2 N((K-1) M-N)$ is the real dimension of $\mathcal{G}_{(K-1) M, N}$. Therefore from (12), (13) the leakage term can be upper bounded as

$$
L_{i} \leq 2 P \Delta_{i \max }^{2} \leq \frac{8 P}{\left(c 2^{N_{f}}\right)^{\frac{2}{N_{G}}}} .
$$

In order to achieve the same multiplexing gain as in the perfect CSI situation we have to bound the interference leakage with a constant value independent of the transmit power while letting $P \rightarrow \infty$. From (14), it is obvious that this is achieved if $2^{\frac{2 N_{f}}{N_{G}}}$ scales linearly with $P$. This implies that the number of bits required to bound the power of interference leakage is given by

$$
N_{f}=\frac{N_{G}}{2} \log P=N((K-1) M-N) \log P .
$$

Therefore, after applying receive filter $\mathbf{G}_{i}^{\mathrm{H}}$ at receiver $i$, from (1) we have

$$
\overline{\mathbf{y}}_{i}=\overline{\mathbf{H}}_{i i} \mathbf{x}_{i}+\overline{\mathbf{n}}_{i}
$$


in which $\overline{\mathbf{y}}_{i}=\mathbf{G}_{i}^{\mathrm{H}} \mathbf{y}_{i}, \overline{\mathbf{H}}_{i i}=\mathbf{G}_{i}^{\mathrm{H}} \mathbf{H}_{i i} \mathbf{V}_{i}$ and $\overline{\mathbf{n}}_{i}=\mathbf{G}_{i}^{\mathrm{H}} \mathbf{n}_{i}+\mathbf{e}_{i}$ which has a bounded power independent of $P$. It is clear that there will be no inter-stream interference at each receiver since the channel $\mathbf{H}_{i i}$ and therefore $\overline{\mathbf{H}}_{i i}$ is perfectly known at receiver $i$ (assuming that the alignment solution is provided to the receiver).

It should be noted that the receive filter $\mathbf{G}_{i}^{\mathrm{H}}$ can induce a reduction in the power of the signal of interest. However this will not affect the multiplexing gain, since it can be shown that $\left\|\overline{\mathbf{H}}_{i i}\right\|_{F}^{2}$ is proportional to $\left\|\mathbf{F}_{i}^{\mathrm{H}} \hat{\mathbf{F}}_{i}\right\|_{F}^{2}$ and this term can be bounded below as

$$
\left\|\mathbf{F}_{i}^{\mathrm{H}} \hat{\mathbf{F}}_{i}\right\|_{F}^{2}=N-d_{c}^{2}\left(\hat{\mathbf{F}}_{i}, \mathbf{F}_{i}\right) \geq N-\Delta_{i \max }^{2},
$$

and the desired power is written as

$$
\mathrm{E}\left(\operatorname{tr}\left(\overline{\mathbf{H}}_{i i} \mathbf{x}_{i} \mathbf{x}_{i}^{\mathrm{H}} \overline{\mathbf{H}}_{i i}^{\mathrm{H}}\right)\right)=P\left\|\overline{\mathbf{H}}_{i i}\right\|_{F}^{2} .
$$

Since $\Delta_{i_{\max }}^{2}$ scales with $P^{-1}$, the reduction in the desired signal power will be a constant value independent of $P$. Therefore we can conclude that the desired signal power is of the order of $P$. Furthermore, it is clear from the definition of the $d \times d$ matrix $\overline{\mathbf{H}}_{i i}$ that it is almost surely full rank, since $\mathbf{H}_{i i}$ is independent of $\mathbf{G}_{i}^{\mathrm{H}}$ and $\mathbf{V}_{i}$. Therefore, we conclude that our scheme can achieve $d$ DoF per user if the quantized CSI scales according to (15).

\section{Simulation Results}

In this section, the performance of the proposed scheme is evaluated through numerical simulations. The performance metric is the sum rate evaluated through Monte-Carlo simulations employing the precoders designed using interference alignment.

The achievable sum rate of the MIMO IC using interference alignment precoders under the assumption that the input signals are Gaussian can be written as

$$
\begin{gathered}
R_{\text {sum }}=\sum_{i=1}^{K} \log \left|\sigma^{2} \mathbf{I}_{N}+\sum_{j=1}^{K} \mathbf{H}_{i j} \mathbf{V}_{j} \mathbf{V}_{j}^{\mathrm{H}} \mathbf{H}_{i j}^{\mathrm{H}}\right| \\
-\sum_{i=1}^{K} \log \left|\sigma^{2} \mathbf{I}_{N}+\sum_{j=1, j \neq i}^{K} \mathbf{H}_{i j} \mathbf{V}_{j} \mathbf{V}_{j}^{\mathrm{H}} \mathbf{H}_{i j}^{\mathrm{H}}\right| .
\end{gathered}
$$

A three-user IC with two antennas per node and one data stream for each transmitter is considered. Entries of the channel matrices are generated according to $\mathcal{C N}(0,1)$ and the performance results are averaged over the channel realizations. Our proposed method is compared to the naive method where the interfering channel matrices toward each receiver are independently vectorized and quantized based on the idea of composite Grassmann manifold [9] and finally the indices of the quantized vectors are fed back to the transmitters (denoted by "Naive" in the figures).

Figure 1 shows the achievable sum rate versus transmit SNR for $N_{f}=5$ and 10 feedback bits when the precoders are designed based on the quantized feedback. Instead of the optimal subspace packing codebook, random vector

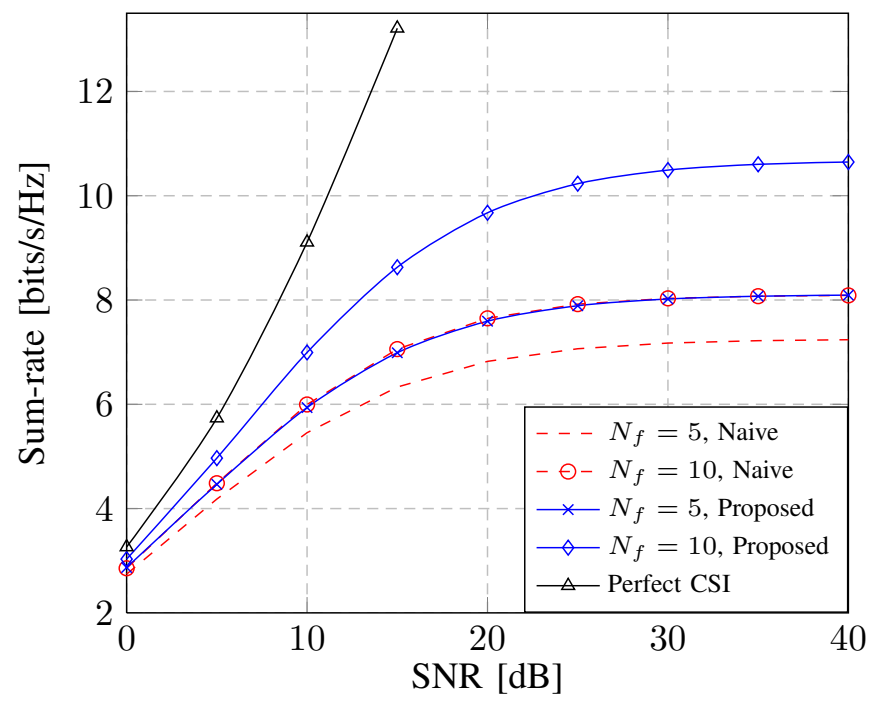

Fig. 1. Performance comparison of quantization methods, for 3-user MIMO IC, $N=2, M=2$ using (19).

quantization codebook is used where in our proposed method the codebook entries are independent $(K-1) M \times N$ random truncated unitary matrices generated from the Haar distribution. For the naive method, random unit norm vectors are used in the codebook construction.

The sum rate in (19) is achievable when optimum receivers (not including the projection filters $\mathbf{G}_{i}^{\mathrm{H}}$ ) are used at the receivers. Since the achievable scheme in Section IV is using the projection filters $\mathbf{G}_{i}^{\mathrm{H}}$, we evaluated the performance achieved by this scheme as well, defined as

$$
\begin{gathered}
R_{\text {sum }}^{\prime}=\sum_{i=1}^{K} \log \left|\sigma^{2} \mathbf{G}_{i}^{\mathrm{H}} \mathbf{G}_{i}+\sum_{j=1}^{K} \mathbf{G}_{i}^{\mathrm{H}} \mathbf{H}_{i j} \mathbf{V}_{j} \mathbf{V}_{j}^{\mathrm{H}} \mathbf{H}_{i j}^{\mathrm{H}} \mathbf{G}_{i}\right| \\
-\sum_{i=1}^{K} \log \left|\sigma^{2} \mathbf{G}_{i}^{\mathrm{H}} \mathbf{G}_{i}+\sum_{j=1, j \neq i}^{K} \mathbf{G}_{i}^{\mathrm{H}} \mathbf{H}_{i j} \mathbf{V}_{j} \mathbf{V}_{j}^{\mathrm{H}} \mathbf{H}_{i j}^{\mathrm{H}} \mathbf{G}_{i}\right| .
\end{gathered}
$$

Results are provided in Figure 2.

The slope of the curves on Figure 2 at high SNR gives us an indication of the DoF. It is clear from Figure 2 that the slope of the rate curve with quantized feedback matches that of perfect CSI when the number of feedback bits is scaled according to (15). We have used $N_{f}=[0,7,13,20,26]$ bits and their corresponding powers $\left(P=2^{\frac{2 N_{f}}{N_{G}}}\right)$ to generate the curve which exhibits the achievable DoF. Simulations were performed only up to SNR of $20 \mathrm{~dB}$ due to the complexity associated to the exponential size of the codebook.

From both figures, it is clear that the performance improves with the number of feedback bits, and that the proposed method outperforms the naive method.

\section{CONCLUSION}

A new CSI feedback scheme for interference alignment on the K-user MIMO interference channel was proposed consist- 


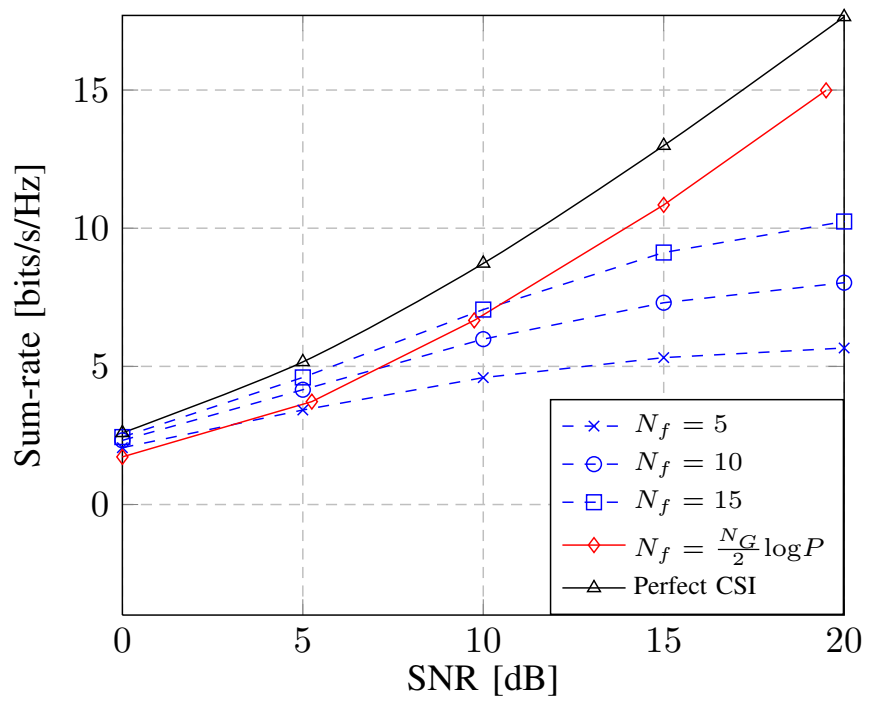

Fig. 2. Performance according to (20) of the proposed method for different number of bits, for 3-user MIMO IC, $N=2$, $M=2$

ing in a parsimonious representation based on the Grassmann manifold. We characterized the proper scaling of number of feedback bits with transmit power in order to preserve the multiplexing gain achievable using perfect CSI. Simulations results confirm that our scheme provides a better sum rate performance compared to the naive feedback scheme for the same number of feedback bits.

\section{ACKNOWLEDGMENT}

This work was supported by the FP7 project HIATUS (grant \#265578) of the European Commission and by the Austrian Science Fund (FWF) through grant NFN SISE (S106).

\section{REFERENCES}

[1] V. Cadambe and S. Jafar, "Interference alignment and the degrees of freedom of the $\mathrm{K}$ user interference channel," IEEE Trans. Inf. Theory, vol. 54, no. 8, pp. 3425-3441, Aug. 2008.

[2] T. Gou and S. A. Jafar, "Degrees of freedom of the $K$ user $M \times N$ MIMO interference channel," IEEE Trans. Information Theory, vol. 56, no. 12, pp. 6040-6057, Dec. 2010.

[3] D. J. Love, R. W. Heath, Jr, V. K. N. Lau, D. Gesbert, B. Rao, and M. Andrews, "An overview of limited feedback in wireless communication systems," IEEE Journal on Selected Areas in Communications, vol. 26, no. 8, pp. 1341-1365, Oct. 2008.

[4] D. J. Love and R. W. Heath, Jr, "Limited feedback unitary precoding for spatial multiplexing systems," IEEE Trans. Inf. Theory, vol. 51, no. 8, pp. 2967-2976, Aug. 2005

[5] W. Dai, Y. Liu, and B. Rider, "Quantization bounds on Grassmann manifolds and applications to MIMO communications," IEEE Trans. Inf. Theory, vol. 54, no. 3, pp. 1108-1123, Mar. 2008.

[6] A. Barg and D. Y. Nogin, "Bounds on packings of spheres in the Grassmann manifold," IEEE Trans. Inf. Theory, vol. 48, no. 9, pp. 24502454, Sep. 2002.

[7] W. Santipach and M. L. Honig, "Capacity of a multiple-antenna fading channel with a quantized precoding matrix," IEEE Trans. Inf. Theory, vol. 55 , no. 3, pp. $1218-1234$, Mar. 2009

[8] J. Thukral and H. Bolcskei, "Interference alignment with limited feedback," in Proc. IEEE Int. Symp. Information Theory (ISIT), Seoul, Jun. 2009.

[9] R. T. Krishnamachari and M. K. Varanasi, "Interference alignment under limited feedback for MIMO interference channels," in Proc. IEEE Int. Symp. Information Theory (ISIT), Austin, TX, Jun. 2010.

[10] R. Tresch and M. Guillaud, "Cellular interference alignment with imperfect channel knowledge," in Proc. IEEE International Conference on Communications (ICC), Dresden, Germany, Jun. 2009.

[11] J. Kim, S. Moon, S. Lee, and I. Lee, "A new channel quantization strategy for MIMO interference alignment with limited feedback," IEEE Trans. Wireless Commun., vol. 11, no. 1, pp. 358-366, Jan. 2012

[12] C. M. Yetis, T. Gou, S. A. Jafar, and A. H. Kayran, "On feasibility of interference alignment in MIMO interference networks," vol. 58, no. 9, Sep. 2010.

[13] M. Razaviyayn, G. Lyubeznik, and Z.-Q. Luo, "On the degrees of freedom achievable through interference alignment in a MIMO interference channel," Apr. 2011, preprint, http://arxiv.org/abs/1104.0992.

[14] G. Bresler, D. Cartwright, and D. Tse, "Settling the feasibility of interference alignment for the MIMO interference channel: the symmetric square case," Apr. 2011, preprint, http://arxiv.org/abs/1104.0888.

[15] R. T. Krishnamachari, A Geometric Framework for Analyzing the Performance of Multiple-Antenna Systems under Finite-Rate Feedback. $\mathrm{PhD}$ thesis, University of Colorado, Boulder, 2011. 